\title{
Quality systems in health care: a sociotechnical approach
}

\author{
P.P.M. Harteloh * \\ Department of Health Policy and Management, Erasmus University Rotterdam, P.O. Box 1738, 3000 DR Rotterdam, The Netherlands
}

Received 7 March 2002; accepted 29 September 2002

\begin{abstract}
In this paper, we explore a sociotechnical approach to construct quality systems as an alternative to the traditional, ISO orientated approach. A sociotechnical approach is characterised as bottom-up, incremental, information technology facilitated and indicator driven. Its purpose is to ground quality assurance in medical practice and to provide meaning to those directly involved (patients, health care providers and medical professionals). Meaning depends on information. According to contemporary theory of meaning, facts become information on quality if the structure of data represents the structure of the quality concept. The structure of the quality concept is exemplified by definitions of the quality of care, most of them comparing actual properties of care with requirements, expectations, standards or guidelines. So, raw data or measurements have to be compared with a normative frame of reference in order to become information on the quality of care. Quality indicators conceptualise this theory of meaning. Therefore, constructing quality systems by developing quality indicators is important for the meaning of quality assurance in health care. It makes a system to a quality system and suits a sociotechnical approach by grounding the formal structure of the system in a social reality.
\end{abstract}

(C) 2002 Elsevier Science Ireland Ltd. All rights reserved.

Keywords: Quality systems; Quality assurance; Quality indicators; Theory of meaning; Information; Sociotechnical approach

\section{Introduction}

'Observation tells us that every state is an association, and that every association is formed with a view to some good purpose. I say 'good' because in all their actions all men do in fact aim at that what they think good. Clearly then all associations aim at some good, ...' Aristotle, Politics, 1252a1-4

\footnotetext{
* Tel.: +31-10-408-8541
}

E-mail address: harteloh@bmg.eur.nl (P.P.M. Harteloh).
Quality assurance is a relatively new discipline in health care. Its aim is to facilitate the delivery of good medical care. It deals with topics, such as the effectiveness, efficiency and the access of health care, the values and satisfaction of patients, the attitude of health care workers, and problems such as medical errors and practice variations [1]. Medical audit and peer review are its traditional methods [2]. These methods enable medical professionals to analyse problems in a systematic way and to reduce practice variations by developing and using guidelines or standards. By gathering data on the effect of one's own medical actions, the medical professional continuously improves and 
can become a master in the technique and art of medicine [3]. Quality systems are more and more used to integrate and facilitate the different methods for quality assurance and to create a formal mechanism for internal feed back in health care organisations and external accountability to patients, providers and society [4]. In this paper, we focus on the construction of quality systems. We will address the question 'what makes a system to a quality system?' For answering this question we explore systems theory on the one hand and the meaning of 'quality' on the other. As meaning is closely linked with information, the role of information in constructing quality systems will be elaborated on. A formal and a social construction of a quality system will be distinguished. An integral application of both aspects could be called a sociotechnical approach for constructing quality systems in health care organisations. In this paper, we will define such a sociotechnical approach and delineate its role in giving meaning to quality assurance in health care.

\section{The meaning of quality systems in health care}

The (explicit) quest for quality of care originates in the early 20th century [5]. It deals with medical errors and practice variations and finds a paradigm in the work of the surgeon Codman, who tried to control and improve his (own) medical practice by a systematic evaluation of its outcomes. The search for mechanisms to control medical practice is a leading theme ever since. Medical audit and peer review are its modern appearance from a professional point of view, and quality systems from an organisational point of view.

A central axiom of systems theory is that reality consists of a set of separate elements (entities) and relationships between them [6]. These relationships can be described in terms of control mechanisms. Most fundamental are feed forward and feed back. Feed forward means (re)acting on the (measurement of) input. Anticipation and adjustment of the health care process to expectations of patients would be an example of feed forward in health care. Feed back means (re)acting on (measurement of) results or output. Adjustment of the health care process in order to improve patient satisfaction rates would be an example of feed back in health care. Both mechanisms can be combined to complex control mechanisms which keep up the internal equilibrium of the system by managing a continuous flow of input and output (patients). The equilibrium is governed by values as standards for good quality care, such as the age old 'charitas' in nursing, the effectiveness and efficiency in modern (evidence based) medical practice, or the autonomy and wellbeing of patients in society.

In order to be a quality system, a system has to represent the meaning of the quality concept. But what is the meaning of 'quality'? From a pragmatic point of view 'meaning is use' [7]. So, the meaning of quality in health care can be found by studying the use of the word 'quality' in the debate on the quality of care. Nowadays, this debate shows an increasing influence of industrial ideas about quality management [8]. Quality is no longer considered a (static) property of the health care system (effectiveness, efficiency), but more and more a (dynamic) capacity of medical work [9], e.g. a performance of health care providers in their interaction with patients. In general, judging the quality of care means comparing actual properties of care with professional requirements and with expectations of patients or society. Patients will focus on health gains, satisfaction or wellbeing on the one hand and on their wants, needs and expectations on the other, the medical professional will judge the effect of medical interventions against a background of scientific knowledge of its possibilities, the manager of health care organisations will focus on the costs and the number of interventions delivered in relation to the available materials and manpower. Patients, medical professionals and managers judge quality in the same way. However, they differ in their focus on specific aspects of medical care on the one hand and in their expectations on the other. In general, the word 'quality' is used when there is an optimal balance between possibilities realised and a framework of norms and values, considering an aspect of health care [10,11]. This rule is a formal criterion of meaning. Speakers use a rule for sending a message, listeners interpret the message according to certain rules. If the rule used by the 
listener, complies with the rule used by the speaker, information is transmitted. In choosing a rule for interpretation, speaker and listener are free, however, not in following a rule. The speaker can (only) check correct understanding of a word through observation of the way listeners follow the rule, intended by the message. The listener can only understand the message by following the rule according to which she interprets the message. Rule following is the ground for meaningful transfer of information [7]. Therefore, the rule according to which quality is interpreted, contains its meaning. Explicit application of this (semantic) rule is what makes $a$ system to a quality system. In constructing a quality system a formal and a social application of this rule can be distinguished.

\section{The formal construction of a quality system}

The formal construction of a quality system requires a description of its elements and the relationships between them, e.g. developing a model. Incorporating the meaning of quality in this construction makes the system a quality system. The ISO-9000 is the framework for constructing quality systems (Table 1). It defines a quality system as 'the organisational structure, responsibilities, procedures, processes, and resources to assure and improve quality' [12]. It provides a blue print of an excellent organisation. This blueprint is basically the same for most quality systems. It appears in the ISO 9000:2000 standard for quality systems, the European Foundation for Quality Management (EFQM) model for business excellence, and in the concept of Total Quality Control. From a conceptual point of view, 'rational control' seems to be a central value. ${ }^{1}$ The idea is that variations in the primary process have to be controlled in order to adjust the outcome of the process to the needs and demands of clients

\footnotetext{
1 'A phenomenon will be said to be controlled when, through the use of past experience, we can predict, at least within limits, how the phenomenon may be expected to vary in the future. Here it is understood that prediction means that we can state, at least approximately, the probability that the observed phenomenon will fall within the given limits' [13].
}

(patients) and to professional requirements. Rational control is a reflexive process, exemplified by the Deming circle, an ongoing sequence of 'plando-check-(re)act' (Fig. 1). A reflexive attitude of health care workers, peer review and meeting in quality circles are its social appearance. These requirements for rational control determine the construction of a quality system in health care organisations [14].

Several elements facilitate rational control in health care organisations. Leadership is necessary to legitimize, facilitate and stimulate quality assurance. Policy gives sense by applying concepts and reason to actions or forming a plan for improvement (plan). For the actual control of the primary process, health care workers need practical knowledge of quality assurance techniques, such as statistical process control, Pareto analysis, Ishikawa diagrams, etc. Therefore training and education are required. In a service industry, like health care, the primary process as a focus of quality assurance has to be made explicit by guidelines, standards and protocols. Standards and guidelines contain the normative framework of the primary process, protocols describe its actual realisation. They can be used to monitor the execution of the primary process $(d o)$. Measurements and data inform health care workers, providers and patients about the actual properties of the primary process (check). This information can be collected in a data (knowledge) base. The (raw) data in such a knowledge base becomes information on quality by applying a semantic rule in such a way that data exemplify the meaning of quality. Reactions on this information establish a feed forward or feed back mechanism to control the primary process in organisations.

Rational control is again a leading principle when the satisfaction of patients or health care workers and social wellbeing are seen as the most important outcomes of a quality system. In general, only a controlled process will be able to meet the needs and demands of patients. Being in control of one's own work gives the health care worker a feeling of satisfaction, and a controlled process contributes to social wellbeing in contrast to random disorder. 
Table 1

Constructing quality systems in health care

\begin{tabular}{lll}
\hline & The formal (ISO) orientated approach & Sociotechnical approach \\
\hline Theoretical basis & General systems theory & A theory of meaning \\
Purpose & $\begin{array}{l}\text { External accountability by standardisation and } \\
\text { certification }\end{array}$ & $\begin{array}{l}\text { Stimulating and facilitating internal reflexivity } \\
\text { of the organisation }\end{array}$ \\
Construction (by) & $\begin{array}{l}\text { Describing elements and their formal relationships } \\
\text { (External) blue print }\end{array}$ & $\begin{array}{l}\text { Dialogue } \\
\text { Need of health care workers and patients }\end{array}$ \\
& $\begin{array}{l}\text { Organisational diagnosis } \\
\text { Stepwise strategy, according to a plan or blue print }\end{array}$ & $\begin{array}{l}\text { Incremental strategy, according to politics or } \\
\text { social dynamics }\end{array}$ \\
& & Bottom-up \\
Implementation & Top down & Continuous growth \\
& $\begin{array}{l}\text { Project, scheduled in time } \\
\text { Standard driven }\end{array}$ & Indicator driven \\
& Facilitated by education and training & Guided by qualitative research and participation \\
Guided by external advise & Quality as (dynamic) capacity \\
Meaning & Quality as a (static) property & Focus on information \\
& Focus on data & Social interpretation (Quality circles)
\end{tabular}

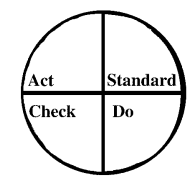

Quality

as ses sm ent

(Donabe dian)
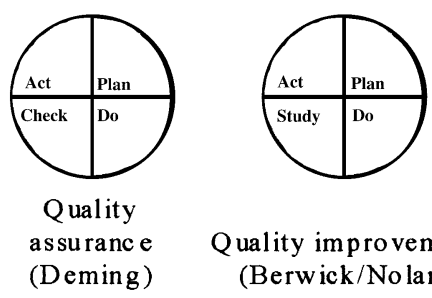

Quality improvement (Berwick/Nolan)
Fig. 1. Varieties of the Deming circle.

In this way, rational control becomes guiding principle and blue print of a quality system (Fig. 2).

The formal relationships between the elements of a quality system are formed by a flow of information. Therefore, information technology (IT) is an important (material) focus of a quality system [15]. Medical professionals are responsible for the intrinsic quality of the primary process. They need information in order to control medical care and realise its valued properties [16]. Patients and society need information in order to judge if their expectations are met and public means are spent well. According to contemporary theory of meaning, data becomes information on quality if the structure of the data represents the structure of the quality concept. This structure is exemplified by definitions of the quality of care, comparing actual properties of care with requirements or expectations. Thus, data representing medical events (e.g. counting readmissions or patient satisfaction measurements) have to be compared with a normative frame of reference (guidelines, standards, norms, values) in order to be interpreted in terms of quality. Quality indicators conceptualise this meaning theory (Fig. 3). A norm or value is added to (raw) data, e.g. avoidable mortality, unexpected readmissions, or not more than 2 standard deviations from the mean patient satisfaction percentage of comparable health care organisations. In this way, quality indicators transfer the meaning of the quality concept.

Quality indicators are the application of a semantic rule, through which data becomes information on quality. Together with data which (directly) represents aspects of the primary process, quality indicators could be sampled in a central database from which information flows to medical professionals or to external stakeholders (providers, patients and society), realising internal reflexivity and external accountability (Fig. 4). Only a well balanced set of indicators can fulfil both functions. Therefore, its construction re- 


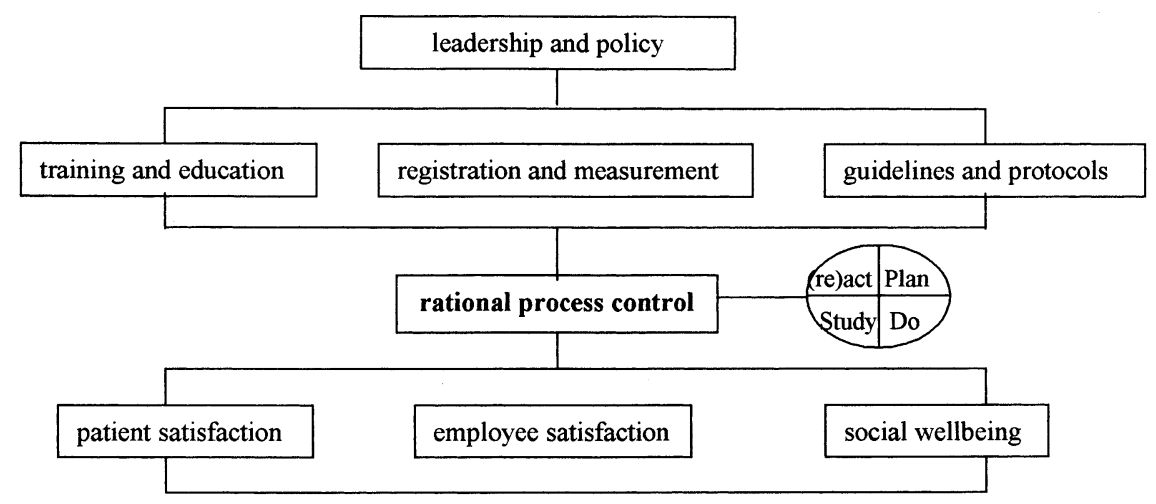

Fig. 2. The elements of a quality system.

quires participation of all parties involved (medical professionals, patients, managers, providers), a well structured dialogue between them, and a carefully designed interpretation process of data and indicators.

\section{The social construction of a quality system}

The formal construction of a quality system requires adequate social interactions to become fully operational in a health care organisation. The wants, needs and demands of patients have to be made explicit and used as input for the feed back or feed forward processes in quality systems in order to translate them into characteristics of medical care and health care organisations. Policy, politics and (scientific) knowledge of patients all play an important role in the construction of a quality system. Together with the development of evidence based guidelines or standards and peer review techniques they form the traditional body of knowledge of quality assurance as a managerial discipline.

The traditional body of knowledge for constructing quality systems stems from industry. Ideas of its American founding fathers, Deming and Juran, developed in Japanese industry to

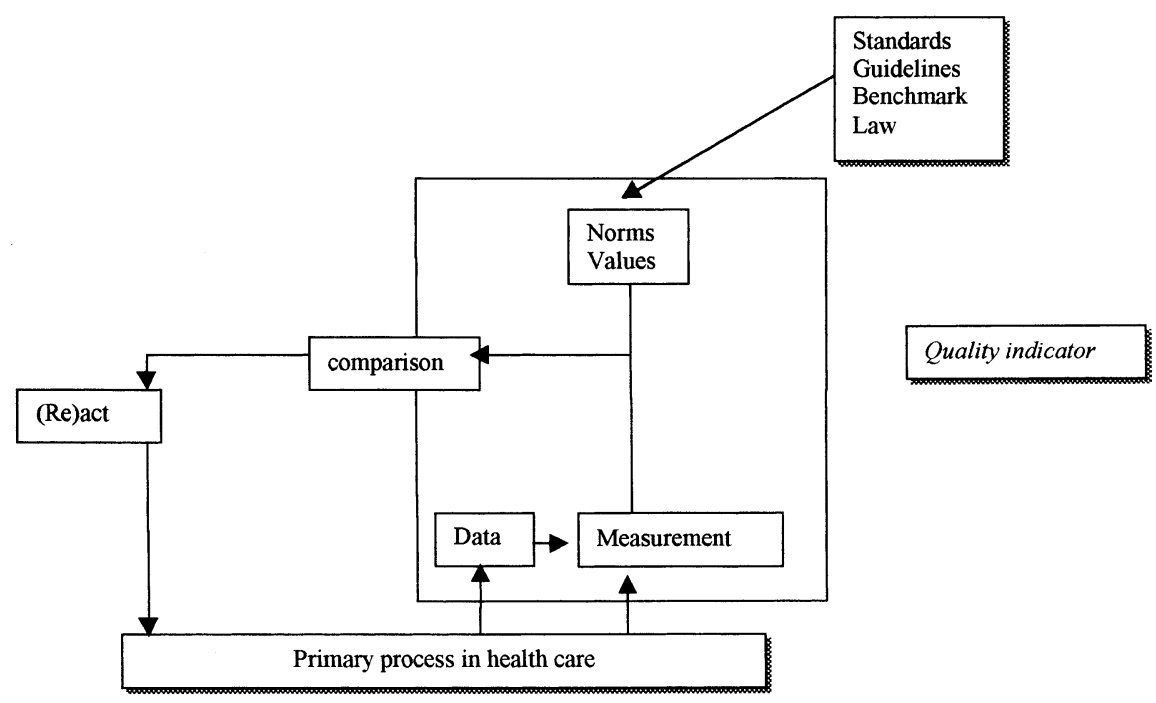

Fig. 3. Conceptual model of a quality indicator. 


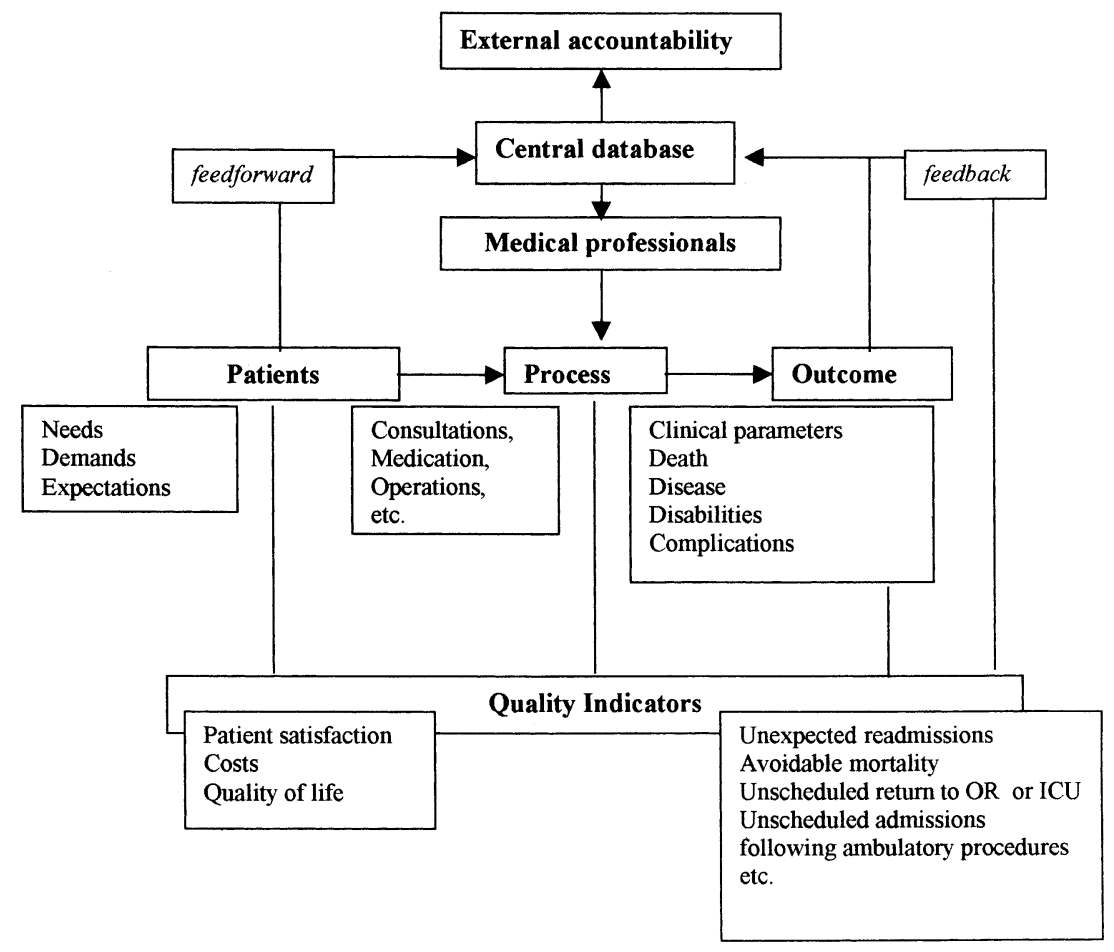

Fig. 4. The relationships of a quality system.

'Total Quality Management' (TQM), an organisational wide reflexive attitude and pursuit of continuous quality improvement (CQI). In the early 1990s these ideas gained ground in Western health care [17]. TQM and CQI were promoted as an alternative for bureaucracy, and as a cure for practice variations, medical errors and ever increasing costs in health care $[18,19]$. TQM stimulates the construction of quality systems to involve the whole organisation in CQI.

The industrial approach is often questioned in health care. It is considered too formal in nature, restricted to the structure of medical work, and not adapted to the medical professionals and the specific requirements for quality assurance in health care, such as professional responsibility and communication [20]. Moreover, Øvretveit noticed that TQM has not been adopted in Japanese health care [21]. In Japan, a bottom-up introduction of quality methods based on quality circles (multidisciplinary groups of employees reflecting on their work) is considered more suitable for quality assurance in health care [21]. So we should be careful to copy industrial principles of quality assurance to health care.

The industrial principles of quality assurance rest on general systems theory. As we have seen, general systems theory involves a reduction of reality to elements and relationships between them. This is an axiom, an unproven starting point for an inference. Reality can also be considered as a machine, an organism or a personality [22]. The reduction presupposes an idea about entities and relationships as well. In general systems theory both are considered to be formal in nature. This reduction is perhaps suitable for the construction of bridges, aeroplanes or computers, but for application to health care the social aspect of reality has to be considered as well. This means adaptation of general systems theory to medical discourse, the meaning of quality, the nature of professional practice, and to the (local) needs or demands of patients. A sociotechnical approach can facilitate this adaptation. 
A sociotechnical approach can be based on cultural, historical, ethnographical considerations, or science and technology studies [23]. General aim of a sociotechnical approach is to make sense of a situation [24]. For this, a theory of meaning is required. In constructing quality systems, the semantic rule underlying the quality concept is what makes $a$ system to a quality system. It gives the formal structure of the system. The use of this rule in communication between people forms its social reality. A sociotechnical approach to the construction of quality systems in health care means applying a formal rule, e.g. know-how to follow or use a rule in a social reality.

From a sociotechnical point of view, a quality system is an association, a network of humans and artefacts. Its aim is to promote the delivery of good health care, to facilitate the excellence of the primary process. It manifests itself as rational control, the core value of quality assurance, an essential property of the primary process. A value is part of our social reality. Therefore by reading a quality system in terms of values, the social reveals itself. From a general systems point of view, the construction of a quality system is based on rational theory. From a social point of view, it is a realisation of values. There is a political dimension in its construction. In the implementation of quality systems this political dimension reveals itself. Most important is a general involvement. A clear choice by the Board of Directors for a specific quality model or system, good communication about goals, means and results, involvement of medical professionals, a positive attitude of employees to changes and innovations, and a decentralised policy making all determine successful implementation of a quality system [25]. Motivation has to come from the drive to do the medical work as good as possible. Therefore, the organisation has to be adjusted to the best working practice [26]; not the other way around, e.g. adjust the primary process to a blue print of the 'best' organisation. Incremental actions are needed to give room to local adaptation. A long term plan is needed as a framework for reason. An outline for a strategy in this respect could be called a sociotechnical approach. It differs from a traditional, ISO orientated approach in theoretical basis, purpose, (actual) construction, implementation, and above all in translating the meaning of the quality concept into medical practice (Table 1).

\section{Conclusion}

From a sociotechnical point of view a quality system is an association, a network of humans and artefacts, formed with a view to some good purpose, e.g. improving the quality of medical practice by internal reflexivity of medical professionals in health care organisations and (a valid) external accountability to patients or society. A bottom-up construction suits a sociotechnical approach to quality systems in health care. Quality assurance should be grounded in medical practice and have meaning to those directly involved (medical professionals, patients, managers). Actions can then really aim at improvement, not at (just) controlling the primary process. For constructing quality systems this means (local) exploration of medical practice with a set of quality indicators as minimal outcome. Quality indicators provide the quality system with meaning by comparing actual properties of care with a normative frame of reference. They exemplify the definition of quality. Therefore, constructing a quality system by developing quality indicators is important for the meaning of quality assurance in health care. Research on creating a well balanced set of indicators for screening problems in the primary process of health care, the adherence of medical professionals to protocols, guidelines or standards, the outcomes of medical care, or the performance of medical acts and devices is important for the innovation of quality assurance in health care.

\section{References}

[1] Blumenthal D. The origins of the quality-of-care debate. New England Journal of Medicine 1996;335:1146-9.

[2] Brook RH, McGlynn EA, Cleary PD. Measuring quality of care. New England Journal of Medicine 1996;335:9669.

[3] Chassin MR. Improving the quality of care. New England Journal of Medicine 1996;335:1060-3. 
[4] Institute of Medicine (IOM). Crossing the Quality Chasm. A new health system for the 21st century. Washington: National Academy Press; 2001.

[5] Harvey G. Quality in health care: traditions, influences and future directions. International Journal Quality Health Care 1996;8:341-50.

[6] Bertalanffy VL. General systems theory. New York: Braziller, 1968

[7] Wittgenstein L. Philosophical investigations. Oxford: Basil Blackwell Ltd, 1958.

[8] Harteloh PPM, Verheggen FWSM. Quality assurance in health care: from a traditional towards a modern approach. Health Policy 1994;27:261-70.

[9] Steffen GE. Quality medical care: a definition. JAMA 1988;260:56-61.

[10] Lohr KH, Harris-Wehling JH. Medicare: a strategy for quality assurance, I: a recapitulation of the study and a definition of quality of care. Quality Review Bulletin 1991;17:6-9.

[11] Campbell SM, Buetow RSA. Defining quality of care. Society Science Medicine 2000;51:1611-25.

[12] ISO. International Organisation for Standardisation. ISO 8402. Geneva: ISO; 1994.

[13] Garvin DA. Managing quality. The strategic and competitive edge. New York: The Free Press, 1991:7.

[14] Harteloh PPM. Quality assurance systems in health care: a rational construction. In: The quality of health services in a united Europe: lessons for others. Utrecht: CBO; 1993.

[15] Bates DW, Pappius E, Kuperman GE, et al. Using information systems to measure and improve quality. International Journal of Medical Informatics 1999;53:115-24.
[16] Maxwell CI, Ziegenfuss JT, Chisholm RF. Beyond quality improvement teams: sociotechnical systems theory and self-directed work teams. Quality Management in Health Care 1993;1:59-67.

[17] Merry MD. Total quality management for physicians: translating the new paradigm. Quality Review Bulletin 1990;16:101-5.

[18] Berwick DM, Godfrey AB, Roessner J. Curing health care. San Francisco: Jossey-Bass Publishers, 1991.

[19] Smith R. Medicine's need for Kaizen: putting quality first. British Medical Journal 1990;310:679-80.

[20] Bodenheimer T. The movement for improved quality in health care. New England Journal of Medicine 1999;340:488-92.

[21] Øvretveit J. Japanese healthcare quality improvement. International Journal of Health Care Quality Assurance 2001;14:164-7.

[22] Morgan G. Images of organization. Beverly Hills: Sage Publications, 1986.

[23] Chisholm RF, Ziegenfuss JT. A review of applications of the sociotechnical systems approach to health care organizations. Journal Applied Behaviour Science 1986;22:315-26.

[24] Tonges MC. Work designs: sociotechnical systems for patient care delivery. Nurse Management 1992;23:27-32.

[25] Counte MA, Meurer S. Issues in the assessment of continuous quality improvement implementation in health care organizations. International Journal for Quality in Health Care 2001;13:197-207.

[26] Berg M. Patient care information systems and health care work: a sociotechnical approach. International Journal of Medical Information 1999;55:87-101. 